\title{
IncRNA CYTOR promotes cell proliferation and tumor growth via miR-125b/SEMA4C axis in hepatocellular carcinoma
}

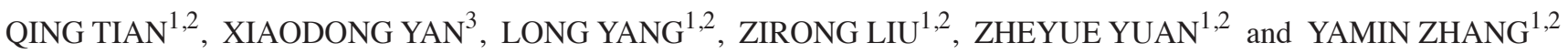 \\ ${ }^{1}$ Department of Hepatobiliary Surgery, Tianjin First Central Hospital, School of Medicine, Nankai University; \\ ${ }^{2}$ Tianjin Key Laboratory for Transplantation; ${ }^{3}$ Department of Hepatobiliary Surgery, First \\ Central Clinic of Tianjin Medical University, Tianjin 300192, P.R China
}

Received December 22, 2020; Accepted May 5, 2021

DOI: $10.3892 / \mathrm{ol} .2021 .13057$

\begin{abstract}
Hepatocellular carcinoma (HCC) is a common malignant tumor worldwide with high morbidity and high mortality rates. Previous studies have demonstrated that cytoskeleton regulator RNA (CYTOR) plays critical roles in the tumorigenesis of various types of cancer. The present study aimed to investigate the clinical significance, biological function and molecular mechanism of CYTOR in the progression of HCC. The expression level of CYTOR was determined by reverse transcription quantitative PCR in HCC tissues and cell lines. The biological function of CYTOR was investigated using CCK-8 assay, EdU immunofluorescence, western blotting and TUNEL assay in vitro. A xenograft tumor model and immunohistochemistry were used to validate the role of CYTOR in vivo. The downstream targets of CYTOR and micro-RNA (miR)-125b were confirmed by RNA immunoprecipitation assay and luciferase reporter assays. The results demonstrated that CYTOR was significantly increased in HCC tissues compared with non-tumor tissues and that CYTOR expression was associated with the poor prognosis of patients with HCC. Furthermore, CYTOR silencing could inhibit the proliferation and promote the apoptosis of HCC cells. CYTOR overexpression had the opposite effects. The results from in vivo xenograft demonstrated that CYTOR knockdown suppressed tumor growth. In addition, CYTOR could directly interact with and negatively regulate miR-125b. Furthermore, semaphorin 4C (SEMA4C) was the target of miR-125b and CYTOR regulated SEMA4C expression by modulating miR-125b. Taken together, the findings from the present study demonstrated that CYTOR could promote cell proliferation and tumor growth by sponging miR-125b and upregulating SEMA4C, which suggested that CYTOR may act as a potential therapeutic target in HCC.
\end{abstract}

Correspondence to: Professor Yamin Zhang, Department of Hepatobiliary Surgery, Tianjin First Central Hospital, School of Medicine, Nankai University, 24 Fukang Road, Tianjin 300192, P.R. China

E-mail: tj_ymzhang@163.com

Key words: cytoskeleton regulator RNA, micro-RNA-125b, hepatocellular carcinoma, cell proliferation, tumor growth

\section{Introduction}

Hepatocellular carcinoma (HCC) is one of the most common hepatic malignancies and remains the leading cause of cancer-associated mortality worldwide $(1,2)$. Surgical resection has been considered as the most effective therapeutic option for patients with HCC; however, it may produce new metastases and promote the growth of existing micro-metastasis (3). It is therefore crucial to determine the molecular mechanisms underlying $\mathrm{HCC}$ and develop novel efficient therapeutic strategies for HCC.

A large part of human genomes cannot encode proteins and most of the transcripts are non-coding RNAs. Long non-coding RNAs (lncRNAs) are defined as highly conserved transcripts of $>200$ nucleotides in length that are not translated into proteins (4). IncRNAs, as novel epigenetic regulatory molecules, are implicated in various cellular and biological processes, and the abnormal expression of lncRNAs are closely associated with tumorigenesis, metastasis and apoptosis by competing for corresponding miRNAs (5). Research on the functional role and molecular mechanisms of lncRNAs have provided a new insight for cancer therapies. Recent studies reported that some lncRNAs are key regulators in HCC $(6,7)$. For example, the expression of the lncRNA SOX9 antisense RNA 1 (SOX9-AS1) is increased in HCC and might have a prognostic significance in patients with HCC. Furthermore, SOX9-AS1 can facilitate HCC progression and metastasis through $\mathrm{Wnt} / \beta$-catenin pathway (8). In addition, the IncRNA DSCAM antisense RNA 1 is upregulated in $\mathrm{HCC}$ and can accelerate the progression of $\mathrm{HCC}$ via sponging micro-RNA (miR)-338-3p (9). A previous study identified the functional lncRNA cytoskeleton regulator RNA (CYTOR), also known as Linc00152, which is situated on human chromosome 2 p11.2 and which abnormal expression is related to the inflammatory response and apoptosis (10). Previous studies have reported that CYTOR plays a pivotal role in the development of various cancers, including colorectal cancer (11), breast cancer (12) and lung cancer (13), in which it functions as an oncogenic lncRNA. However, the biological role and underlying mechanism of CYTOR in HCC cell proliferation and apoptosis remain unknown.

The present study determined CYTOR expression in HCC tissues and its correlation with the clinicopathological char- 
acteristics of patients with HCC. The effects and molecular mechanism of CYTOR on HCC cell proliferation, apoptosis and tumor growth were also investigated. The findings from the present study may accelerate the understanding of HCC pathogenesis and the development of effective therapies.

\section{Materials and methods}

Clinical samples. $\mathrm{HCC}$ tumor tissues $(\mathrm{n}=78)$ and adjacent non-tumor tissues $(n=78)$ were collected from patients with HCC who underwent hepatectomy at Tianjin First Central Hospital between February 2019 and February 2020. The histopathological diagnosis was performed according to the Tumor-Node-Metastasis Staging Guide (14) (based on the extent of the tumor, the extent of spreading to lymph nodes and the presence of metastasis). The samples were immediately frozen and stored at $-80^{\circ} \mathrm{C}$. None of the patients enrolled in this study had received any preoperative chemotherapy or radiotherapy prior to surgery. The clinicopathological characteristics of the patients are described in Table I. All patients have signed written informed consents for the use of their tissues in this research. The protocol was approved by the Clinical Research Ethics Committee of Tianjin First Central Hospital (approval no. 2019N013YY).

The Cancer Genome Atlas (TCGA) program. The gene expression profiles of 180 patients with HCC, including 180 pairs of tumor and adjacent non-tumor tissues, were download from the TCGA dataset (http://www.oncolnc.org/). The survival analysis of CYTOR was visualized by GraphPad Prism version 6.0 software (GraphPad Software Inc.)

Cell lines and cell culture. The normal adult liver epithelial THLE-2 cell line and the human HCC cell lines Huh7, MHCC97-L, MHCC97-H and SK-Hep-1 were obtained from The Cell Bank of Type Culture Collection of The Chinese Academy of Sciences. The cells were cultured in Dulbecco's Modified Eagle's Medium (DMEM; Gibco, CA, USA) supplemented with 10\% FBS (Gibco; Thermo Fisher Scientific, Inc.), $100 \mathrm{mg} / \mathrm{ml}$ streptomycin and $100 \mathrm{U} / \mathrm{ml}$ penicillin (Invitrogen; Merck KGaA) and placed at $37^{\circ} \mathrm{C}$ in a humidified incubator containing $5 \% \mathrm{CO}_{2}$.

Transient transfection. To suppress CYTOR expression, the short hairpin (sh)RNAs targeting CYTOR (sh-CYTOR) were inserted into pLKO.1 plasmid (GenePharma Co., Ltd.) and $4 \mu \mathrm{g}$ sh-CYTOR were transfected into Huh7 cells. Scramble shRNA was used as control (sh-NC). The target sequences of CYTOR shRNAs were as follow: sh\#1: CTGGAAACCTCTTGACTCT and sh\#2: CAGGAAGCTCTATGACACA. To overexpress CYTOR expression, full-length CYTOR cDNA was cloned into pcDNA3.1 plasmid (pcDNA-CYTOR) (GenePharma Co., Ltd.) and then $4 \mu \mathrm{g}$ pcDNA-CYTOR were transfected into Huh7 cells, and the pcDNA3.1 empty vector was used as the control (pcDNA-NC). To regulate miR-125b expression in cells, $50 \mathrm{nM}$ miR-125b mimic (5'-UCCCUGAGACCCUUUAACCUG UGA-3'), $50 \mathrm{nM}$ normal control (NC) mimic (5'-UGACAACCU GGUAGAAAGAGACUUC-3'), $50 \mathrm{nM}$ miR-125b inhibitor (5'-UCCCUGAGACCCUUAACCUGUG-3') and $50 \mathrm{nM} \mathrm{NC}$ inhibitor (5'-UCGGCCUUUUGCUCACAGACCA-3'), designed and synthesized by Shanghai R\&S Biotechnology Co., Ltd., were also transfected into Huh7 cells. Cells were transiently transfected with the above vectors using Lipofectamine ${ }^{\circledR} 2000$ (Invitrogen; Thermo Fisher Scientific, Inc.) according to the manufacturers' protocol at $37^{\circ} \mathrm{C}$. After $48 \mathrm{~h}$ transfection, cells were harvested for further experiments.

$R N A$ extraction and reverse transcription quantitative $(R T-q)$ $P C R$. Total RNA from HCC tissues or Huh7 cells was extracted using TRIzol reagent (Invitrogen; Thermo Fisher Scientific, Inc.). For miRNA analysis, RNA reverse transcription into cDNA was performed using TaqMan MicroRNA Reverse Transcription Kit (Applied Biosystems; Thermo Fisher Scientific, Inc.) and RT-qPCR reactions were performed using TaqMan miRNA assay kit (Applied Biosystems; Thermo Fisher Scientific, Inc.). U6 was used as the internal control. For mRNA analysis, RNA reverse transcription into cDNA was performed using M-MLV reverse transcriptase (Invitrogen; Thermo Fisher Scientific, Inc.) and RT-qPCR reactions were performed with SYBR-Green Real-Time PCR Master Mixes (Thermo Fisher Scientific, Inc.). GAPDH was used as the internal control. The relative expression levels were normalized to endogenous controls and were expressed as $2^{-\Delta \Delta C q}(15)$. The sequences of the primers used were as follows: PCNA, forward, 5'-AACCGG TTACTGAGGGCGAG-3', reverse, 5'-AAAGTCTAGCTGG TTTCGGCT-3'; CYTOR, forward, 5'-AGAATGAAGGCTG AGGTGTG-3',reverse, 5'-CAGCGACCATCCAGTCATTTA-3'; miR-125b, forward, 5'-TCCCTGAGACCCTAACTTGTGA-3', reverse, 5'-AGTCTCAGGGTCCGAGGTATTC-3'; GAPDH, forward, 5'-GAAGGTGAAGGTC GGAGTC-3', reverse, 5'-GAA GATGGTGATGGGATTTC-3'; and U6, forward, 5'-ATTGGA ACGATACAGAGAAGATT-3' and reverse, 5'-GGAACGCTT CACGAATTTG-3'.

Western blotting. Following various transfections, Huh7 cells were lysed in RIPA buffer (Beyotime Institute of Biotechnology) for $10 \mathrm{~min}$ on ice. The concentration of proteins was measured using the BCA method (Beyotime Institute of Biotechnology). Proteins $(50 \mu \mathrm{g})$ were separated by $10 \%$ SDS-PAGE and transferred onto PVDF membranes. Membranes were blocked with 5\% skimmed milk for $2 \mathrm{~h}$ at room temperature and incubated with primary antibodies against Bax (Abcam; cat. no. ab182733; 1:2,000), Bcl-2 (Abcam; cat. no. ab182858; 1:2,000), cleaved-caspase-3 (Abcam; cat. no. ab214430; 1:2,000), pro-caspase-3 (Abcam; cat. no. ab32150; 1:1,000), cleaved-caspase-9 (Abcam; cat. no. ab2324; 1:1,000), pro-caspase-9 (Abcam; cat. no. ab138412; 1:1,000), poly ADP ribose polymerase (PARP) (Cell Signaling Technology, Inc.; cat. no. 9532, 1:1,000), SEMA4C (Abcam; cat. no. ab135856; 1:500) and GAPDH (Cell Signaling Technology, Inc. cat. no. 97166; 1:1,000) overnight at $4^{\circ} \mathrm{C}$. Membranes were then incubated with horseradish peroxidase-labeled secondary antibodies (Abcam; cat. nos. ab205718 and ab205719; 1:2,000) for $2 \mathrm{~h}$ at temperature. Enhanced chemiluminescence reagent (Thermo Fisher Scientific, Inc.) was used to detect the signal on the membrane. The data were analyzed via densitometry using ImageJ software (version 1.8.0; National Institutes of Health) and normalized to expression of the internal control (GAPDH).

Cell Counting Kit-8 (CCK-8) assay. CCK-8 kit assay (Beyotime Institute of Biotechnology) was used to detect cell 
Table I. Association between CYTOR expression and the clinicopathological characteristic of patients with hepatocellular carcinoma.

\begin{tabular}{|c|c|c|c|c|}
\hline \multirow[b]{2}{*}{ Variable } & \multicolumn{4}{|c|}{ CYTOR expression } \\
\hline & Number & Low $(\mathrm{n}=37)$ & $\operatorname{High}(n=41)$ & P-value \\
\hline Age, years & & & & 0.802 \\
\hline$<55$ & 41 & 20 & 21 & \\
\hline$\geq 55$ & 37 & 17 & 20 & \\
\hline Sex & & & & 0.934 \\
\hline Female & 32 & 15 & 17 & \\
\hline Male & 46 & 22 & 24 & \\
\hline Liver cirrhosis & & & & 0.431 \\
\hline No & 60 & 27 & 33 & \\
\hline Yes & 18 & 10 & 8 & \\
\hline Tumor size, $\mathrm{cm}$ & & & & $0.017^{\mathrm{a}}$ \\
\hline$<5 \mathrm{~cm}$ & 46 & 28 & 18 & \\
\hline$\geq 5 \mathrm{~cm}$ & 32 & 9 & 23 & \\
\hline Lymphovascular invasion & & & & $0.026^{\mathrm{a}}$ \\
\hline No & 34 & 21 & 13 & \\
\hline Yes & 44 & 16 & 28 & \\
\hline AFP, ng/ml & & & & 0.838 \\
\hline$<200$ & 41 & 19 & 22 & \\
\hline$\geq 200$ & 37 & 18 & 19 & \\
\hline TNM stage & & & & $0.006^{\mathrm{b}}$ \\
\hline I-II & 56 & 32 & 24 & \\
\hline III-IV & 22 & 5 & 17 & \\
\hline
\end{tabular}

${ }^{\mathrm{a}} \mathrm{P}<0.05$ and ${ }^{\mathrm{b}} \mathrm{P}<0.01$. AFP, $\alpha$ fetoprotein; TNM, Tumor-Node-Metastasis; CYTOR, cytoskeleton regulator RNA.

viability. Briefly, $2 \times 10^{3}$ Huh7 cells were seeded into a 96-well plate. Then, $10 \mu \mathrm{l}$ CCK-8 solution was added in each well at four time points $(24,48,72$ and $96 \mathrm{~h})$ and incubated for another $2 \mathrm{~h}$ at $37^{\circ} \mathrm{C}$. The absorbance was read at $450 \mathrm{~nm}$ on a microplate reader.

EdU immunoflurescence assay. EdU immunoflurescence assay was carried out for detection of cell proliferation. Briefly, Huh7 cells were cultured with EdU solution and incubated for $2 \mathrm{~h}$ at $37^{\circ} \mathrm{C}$. Then, the medium was removed and cells were fixed with PBS containing $4 \%$ paraformaldehyde for $10 \mathrm{~min}$ at room temperature. Subsequently, cells were incubated with $70 \%$ ethanol and stained using the Cell-Light ${ }^{\mathrm{TM}}$ EdU Apollo ${ }^{\circledR} 488$ In Vitro Imaging Kit (RiboBio Co., Ltd.). Cells were visualized using fluorescence microscopy (magnification, x100). EdU analysis was performed using ImageJ software v.1.50 (National Institutes of Health).

Bioinformatics analysis. Putative CYTOR or miR-125b targets were predicted using StarBase version 3.0 (http://starbase.sysu. edu.cn/) or TargetScan (http://www.targetscan.org/vert_60/)

RNA immunoprecipitation (RIP) assay. Following transfection with miR-125b mimic or inhibitor for $48 \mathrm{~h}$, Huh7 cells were lysed with RIPA lysis buffer (Beyotime Institute of Biotechnology) for 10 min on ice following the manufacturers' instructions and incubated with RIP immunoprecipitation buffer mixed with magnetic beads conjugating human anti-Argonaute2 (Ago2) antibody or negative control mouse IgG (EMD Millipore) at $4^{\circ} \mathrm{C}$ overnight. A protein-RNA complex was captured and digested with $0.5 \mathrm{mg} / \mathrm{ml}$ proteinase $\mathrm{K}$ containing $0.1 \%$ SDS to extract RNA. The magnetic beads were repeatedly washed with RIP washing buffer to remove non-specific adsorption as much as possible. RT-qPCR analysis was conducted to estimate the enrichment of CYTOR and miR-125b. Input and IgG served as controls in RIP assay.

Luciferase reporter assay. The wild and mutant type of CYTOR or SEMA4C containing the binding site for miR-125b were cloned into pGL3-reporter vectors, designed as pGL3CYTOR-WT and pGL3-CYTOR-MuT or pGL3-SEMA4C-WT and pGL3-SEMA4C-MuT. Huh7 cells were co-transfected with luciferase reporter vectors and miR-125b mimic, miR-125b inhibitor or negative controls using Lipofectamine 2000. The miR quantities and sequences were as follows: $50 \mathrm{nM}, \mathrm{miR}-125 \mathrm{~b}$ mimic, 5'-UCCCUGAGACCCUUUAACCUGUGA-3'; 50 nM, miR-125b inhibitor, 5'-UCCCUGAGACCCUUAACCUGUG-3'; $50 \mathrm{nM}$, NC mimic, 5'-UGACAACCUGGUAGAAAGAGAC UUC-3'; and 50 nM,NC inhibitor 5'-UCGGCCUUUUGCUCAC AGACCA-3'. After transfection for $48 \mathrm{~h}$, the luciferase activity 
was measured by a Dual-Luciferase Reporter Assay System (Promega Corporation) and normalized to Renilla luciferase activity.

Mice xenograft assay. A total of $36 \mathrm{BALB} / \mathrm{c}$ male nude mice (6-8 weeks old) were purchased from the Animal Center of the Chinese Academy of Science. All animal experiments were performed in the animal laboratory of Tianjin First Central Hospital and the protocol was approved by the Animal Care and Use Committee (approval no. 2019N046YY). Mice were randomly divided into 3 groups of 6 mice as follows: Control group, sh-NC group and sh-CYTOR\#1 group. Huh7 cells stably transfected with sh-CYTOR\#1 or negative control were amplified and a total of $200 \mu \mathrm{l}\left(2.5 \times 10^{7}\right.$ cells $\left./ \mathrm{ml}\right)$ was subcutaneously injected into the dorsal sides of the nude mice. The xenograft tumor size was measured every 7 days for 28 days according to the following formula: Volume $=\left(\right.$ length $\mathrm{x}$ width $\left.{ }^{2}\right) / 2$. Four weeks later, mice were euthanized with $2 \%$ pentobarbital sodium (120 mg/kg bodyweight) and the tumors were collected for immunohistochemistry imaging.

Immunohistochemistry. Tumors were fixed in $10 \%$ formalin (48 h, room temperature), embedded in paraffin and cut into $4 \mu \mathrm{m}$-thick slides. The slides of paraffin-embedded xenograft tissues were probed with specific rabbit anti-SEMA4C antibody (Abcam; cat. no. ab121334; 1:200) and mouse anti-Ki67 (Cell Signaling Technology, Inc.; cat. no. 9449; 1:200) for $48 \mathrm{~h}$ at $4^{\circ} \mathrm{C}$, followed by incubation with the goat anti-rabbit or goat anti-mouse secondary antibodies (Abcam; cat. nos. ab6721 and ab6728; $1: 1,000)$ for $1 \mathrm{~h}$ at $37^{\circ} \mathrm{C}$. Then, the complexes were detected using HRP-streptavidin conjugates, visualized using 3,3'-diaminobenzidine (DAB; Wuhan Boster Biological Technology, Ltd.) and quantified with Image ProPlus (IPP) v7.0 software (Media Cybernetics, Inc.).

Statistical analysis. The data were presented as the means \pm standard deviation of three independent experiments. The differences between two or more groups were analyzed using two-tailed Student's t-tests or one -way ANOVA followed by Turkey's post-hoc test, respectively. Statistical analysis was performed using SPSS Statistics 20.0 software (IBM Corp.) and GraphPad Prism version 6.0 software (GraphPad Software Inc.). The association between CYTOR expression and the clinicopathological characteristics of patients with HCC was analyzed by $\chi^{2}$ test. The correlation between CYTOR expression and miR-125b or SEMA4C expression was evaluated using Pearson's correlation coefficient. Survival analysis was analyzed by the Kaplan-Meier method, followed by difference analysis between the survival curves using log-rank test. $\mathrm{P}<0.05$ was considered to indicate a statistically significant difference.

\section{Results}

CYTOR is upregulated in HCC tissues and associated with the poor survival rate of patients with $H C C$. To investigate the role of CYTOR in HCC, CYTOR expression was evaluated in 78 matched pairs of HCC and adjacent non-tumor tissues by RT-qPCR. The results demonstrated that CYTOR expression was significantly increased in HCC tumor tissues compared with adjacent non-tumor tissues (Fig. 1A). Furthermore,
CYTOR expression was significantly higher in HCC tissues with tumor size $\geq 5 \mathrm{~cm}$ compared with tumor tissues of $<5 \mathrm{~cm}$ (Fig. 1B). In addition, the expression of CYTOR was significantly higher in stage I-II tumors compared with III-IV stage tumors (Fig. 1C). To further confirm the association between CYTOR expression and the clinicopathological characteristics of patients with HCC, the 78 patients were divided into two groups based on the median level of CYTOR in HCC tissues. The results demonstrated that CYTOR exhibited a significant association with tumor size, lymphovascular invasion and Tumor-Node-Metastasis stage (Table I). Kaplan-Meier survival analysis from the TCGA database demonstrated that patients with HCC and low CYTOR expression exhibited a higher overall survival rate compared with patients with high CYTOR expression (Fig. 1D). Consistent with CYTOR expression in HCC tissues, CYTOR expression was significantly increased in HCC cell lines compared with the normal adult liver epithelial THLE-2 cells (Fig. 1E). These findings indicated that CYTOR upregulation was closely associated with the poor prognosis of patients with $\mathrm{HCC}$ and that it may be involved in HCC progression.

CYTOR enhances the proliferation and inhibits the apoptosis of HCC cells. The functional significance of CYTOR in HCC cells was explored. Huh7 cells were selected for subsequent experiments as they displayed the highest CYTOR among all HCC cells. CYTOR was upregulated or downregulated by transfection with pcDNA-CYTOR or shRNA-CYTOR, respectively. The results from RT-qPCR confirmed that CYTOR was effectively overexpressed in Huh7 cells after transfection with pcDNA-CYTOR, while CYTOR was effectively downregulated in Huh7 cells following transfection with shRNA-CYTOR. The sh-CYTOR\#1 was selected for further experiments due to its stronger inhibitory effects compared with sh-CYTOR\#2 (Fig. 2A). The results from CCK-8 assay demonstrated that HCC cell viability was significantly increased in pcDNA-CYTOR transfected cells, whereas it was inhibited in sh-CYTOR\#1 transfected cells compared with control cells (Fig. 2B). Furthermore, CYTOR overexpression could significantly increase the expression of proliferating cell nuclear antigen (PCNA), which is a good indicator of cell proliferation. Conversely, decreasing CYTOR attenuated the proliferation of Huh7 cells compared with control groups (Fig. 2C). Similarly, results from EdU assay indicated that the number of positive cells in pcDNA-CYTOR group was significantly enhanced, whereas it was significantly decreased in the sh-CYTOR\#1 group compared with control groups (Fig. 2D). In addition, transfection with pcDNA-CYTOR significantly increased Bcl-2 expression and decreased the expression of the apoptotic-related proteins, including Bax, cleaved-caspase-3, cleaved-caspase-9 and PARP in Huh7 cells. Transfection with sh-CYTOR\#1 had the opposite effects (Fig. 2E). Taken together, these findings suggested that CYTOR could stimulate the proliferation and attenuate the apoptosis of Huh7 cells.

CYTOR directly interacts with miR-125b. The underlying molecular mechanism of CYTOR on HCC cells was further studied. Starbase prediction algorithm confirmed that miR-125b was a potential binding target of CYTOR (Fig. 3A). Subsequently, 
A

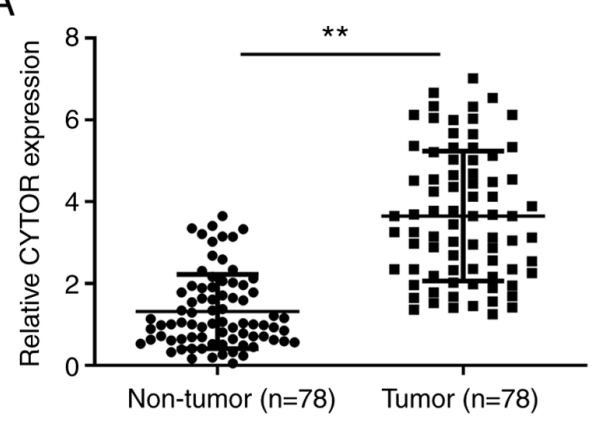

C

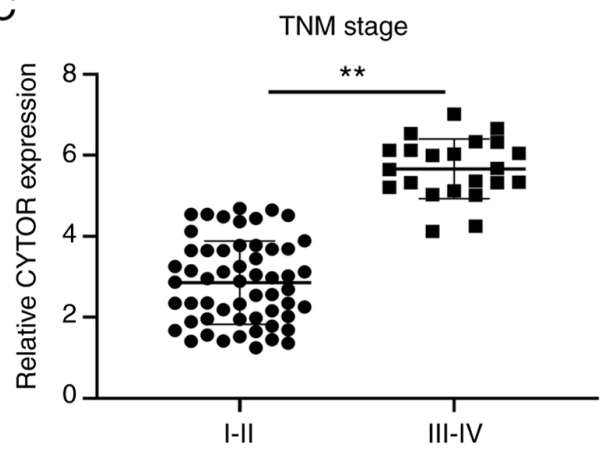

B

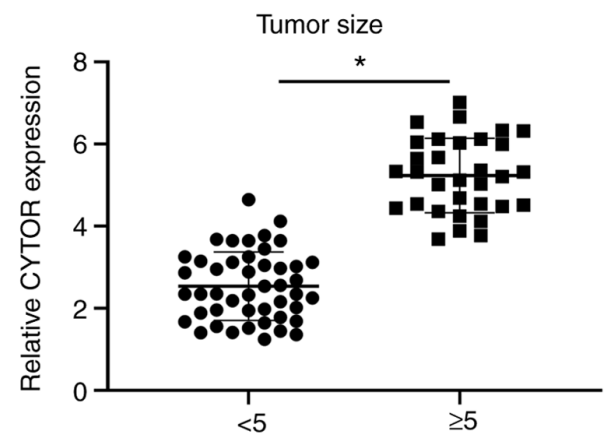

D

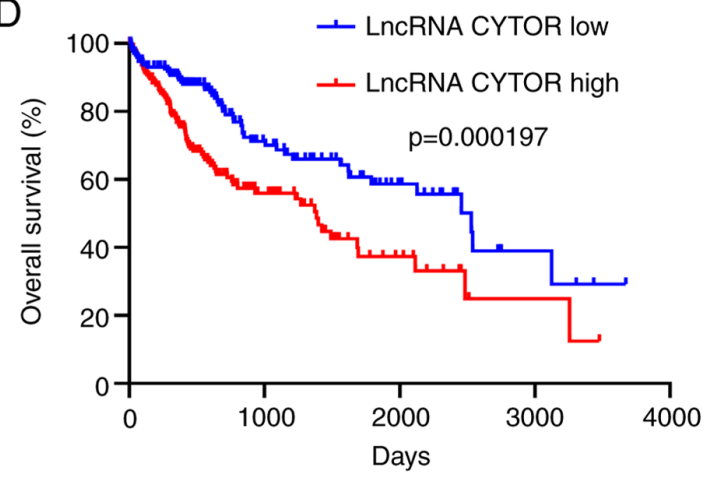

E

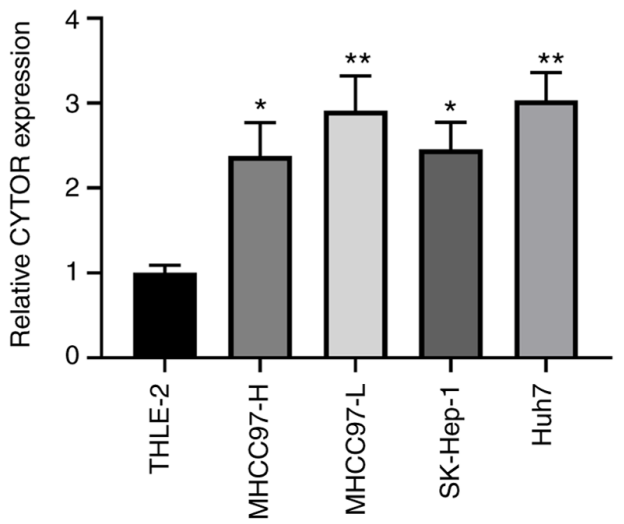

Figure 1. CYTOR is upregulated in HCC and is closely associated with the prognosis of patients with HCC. (A) RT-qPCR detection of CYTOR expression in HCC tumor tissues and matched adjacent non-tumor tissues ( $\mathrm{n}=78$ ). (B) RT-qPCR detection of CYTOR expression in HCC tissues with tumor size $<5 \mathrm{~cm}$ $(\mathrm{n}=46)$ and $\geq 5 \mathrm{~cm}(\mathrm{n}=32)$. (C) RT-qPCR detection of CYTOR expression in stage I-II HCC tissues $(\mathrm{n}=56)$ and stage III-IV HCC tissues $(\mathrm{n}=22)$. (D) Kaplan-Meier analysis of overall survival of patients with HCC and high $(\mathrm{n}=41)$ or low $(\mathrm{n}=37)$ CYTOR expression from The Cancer Genome Atlas database. (E) RT-qPCR detection of CYTOR expression in HCC cell lines and normal adult liver epithelial THLE-2 cell line. ${ }^{*} \mathrm{P}<0.05$ and ${ }^{* *} \mathrm{P}<0.01$. CYTOR, cytoskeleton regulator RNA; RT-qPCR, reverse transcription quantitative PCR; HCC, hepatocellular carcinoma; lncRNA, long non-coding RNA.

miR-125b expression was overexpressed or knocked-down following transfection with miR-125b mimic or inhibitor in Huh7 cells, respectively, and the successful transfection efficiencies were confirmed by RT-qPCR (Fig. 3B). Luciferase reporter assay and RIP assay were subsequently carried out to verify the interaction between CYTOR and miR-125b. The results from luciferase reporter assay demonstrated that miR-125b mimic significantly decreased, while miR-125b inhibitor significantly increased the luciferase activity of CYTOR-WT. However, neither miR-125b overexpression nor suppression had any impact on the luciferase activity of CYTOR-MuT (Fig. 3C). RIP assay revealed that CYTOR and miR-125b expression was elevated in anti-Ago2 group compared with that in control group (Fig. 3D). In addition, the enrichment of CYTOR in anti-Ago2 group was facilitated by miR-125b mimic and inhibited by miR-125b inhibitor (Fig. 3E). Subsequently, RT-qPCR was used to investigate the relationship between CYTOR and miR-125b expression. The results demonstrated that CYTOR overexpression significantly decreased miR-125b expression, whereas miR-125b was markedly increased in sh-CYTOR\#1 group (Fig. 3F), suggesting a negative association between CYTOR and miR-125b expression. Furthermore, miR-125b expression was detected in HCC tissues and the results demonstrated that miR-125b was significantly downregulated in HCC tissues compared with non-tumor tissues (Fig. 3G). Pearson's correlation analysis revealed that CYTOR expression was negatively correlated with miR-125b in HCC tissues (Fig. 3H). These findings suggested that CYTOR may directly interact with miR-125b in HCC.

CYTOR sponges miR-125b to modulate HCC cell proliferation and apoptosis. Whether miR-125b could be involved 

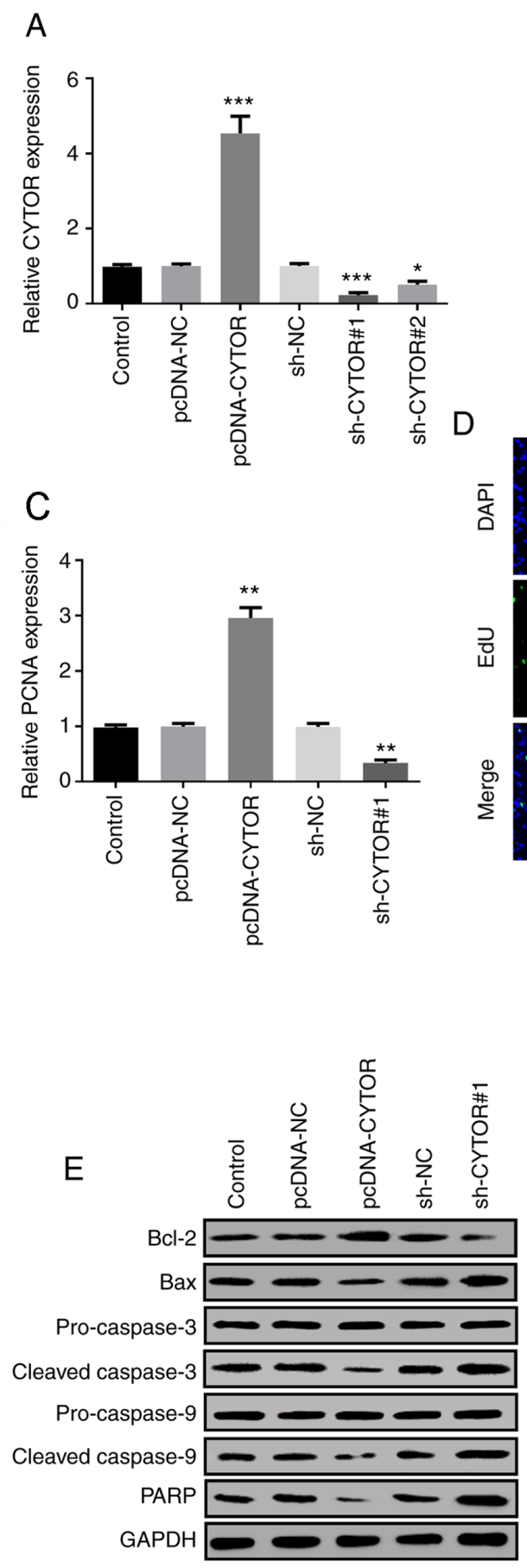

D
B $\rightarrow$ Sh-CYTOR\#1
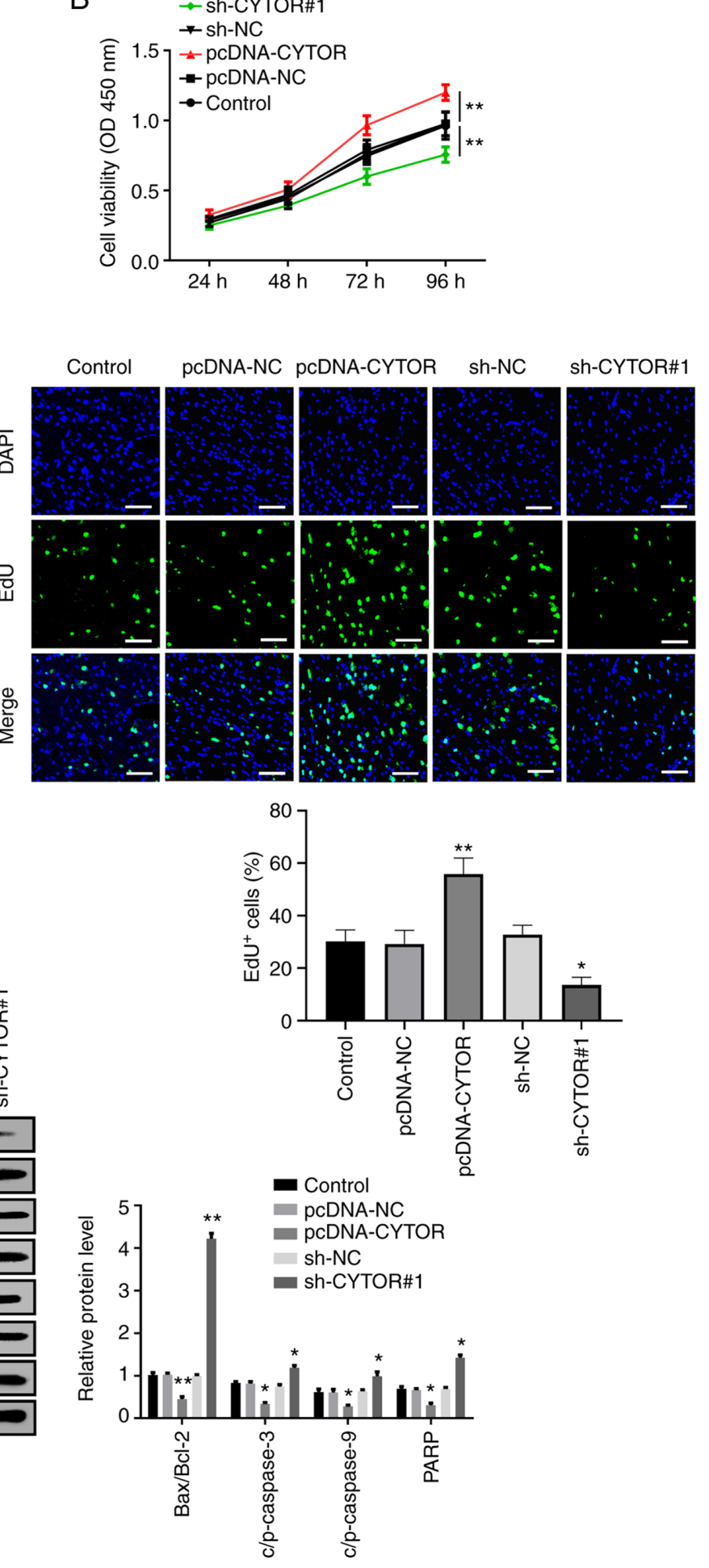

Figure 2.CYTOR promotes the proliferation and inhibit the apoptosis of HCC cells. (A) CYTOR overexpression or knockdown was achieved by pcDNA-CYTOR or CYTOR shRNAs and the transfection efficiency was verified by RT-qPCR. (B) Cell viability was measured by CCK-8 assay. (C) PCNA expression was detected by RT-qPCR and (D) cell proliferative cells measured by EdU assay (scale bar, $20 \mu \mathrm{m}$ ) in Huh7 cells following transfection with pcDNA-CYTOR or sh-CYTOR\#1. (E) Expression of the apoptosis-related proteins Bcl-2, Bax, cleaved/pro-caspase-3, cleaved/pro-caspase-9 and PARP was determined using western blotting. ${ }^{*} \mathrm{P}<0.05,{ }^{* *} \mathrm{P}<0.01$ and ${ }^{* * * *} \mathrm{P}<0.001$. CYTOR, cytoskeleton regulator RNA; RT-qPCR, reverse transcription quantitative PCR; NC, negative control; sh, short hairpin; PARP, poly ADP ribose polymerase; OD optical density.

in the effect of CYTOR on Huh7 cell proliferation and apoptosis was further investigated. Huh7 cells were transfected with sh-CYTOR\#1 alone or with miR-125b inhibitor. The CCK-8 results demonstrated that Huh7 cell viability decrease by sh-CYTOR\#1 could be partly rescued by co-transfection with miR-125b inhibitor (Fig. 4A). The results from RT-qPCR demonstrated that miR-125b inhibitor elevated the inhibitory effect of sh-CYTOR\#1 on HCC cell proliferation (Fig. 4B). Furthermore, the results from Edu assay revealed that the number of posi- 


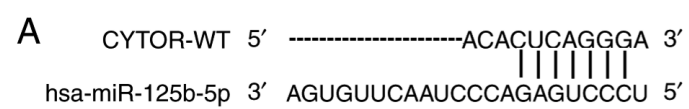

CYTOR-MUT $5^{\prime}$
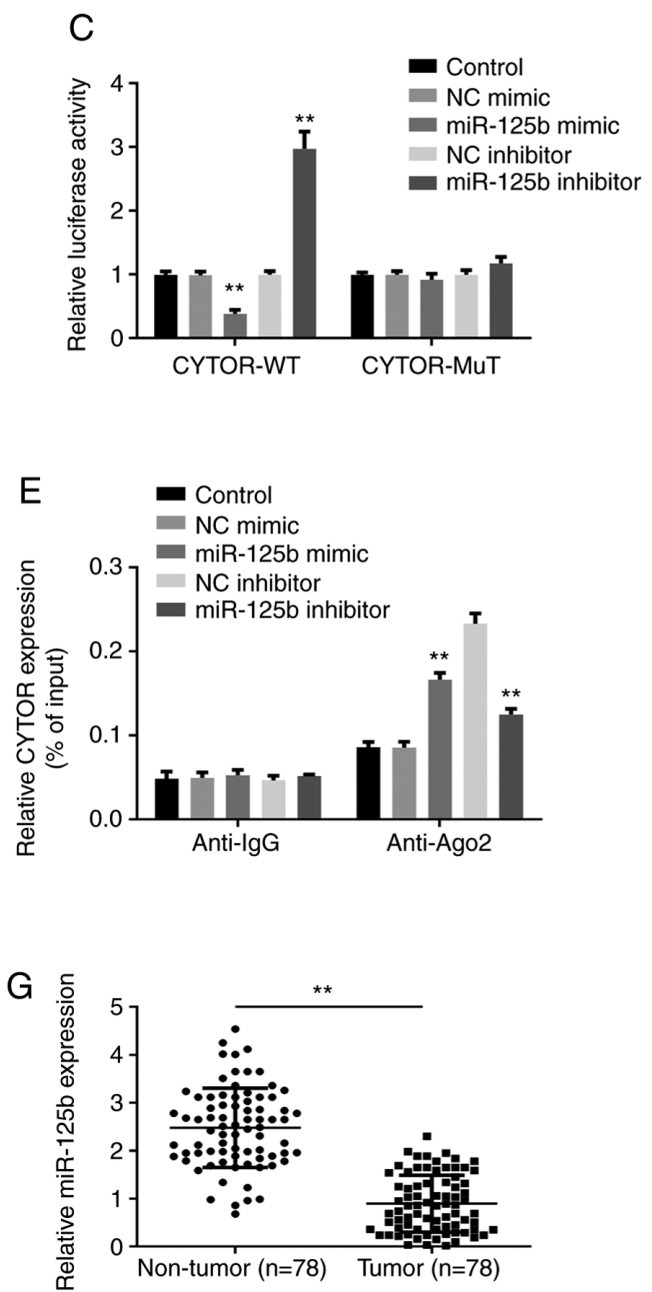

B

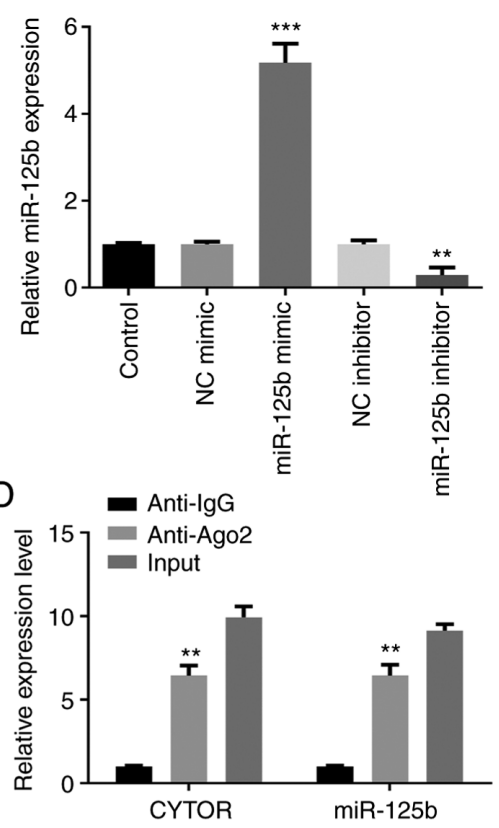

$\mathrm{F}$

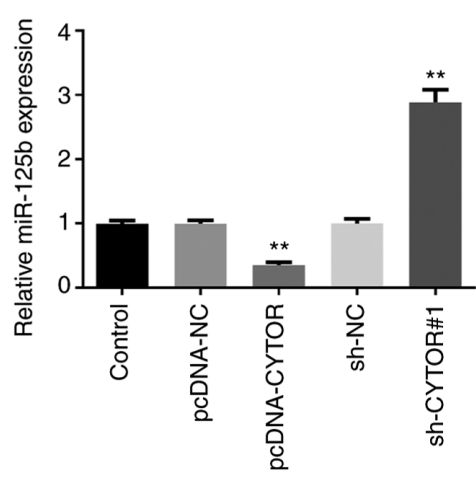

$\mathrm{H}$

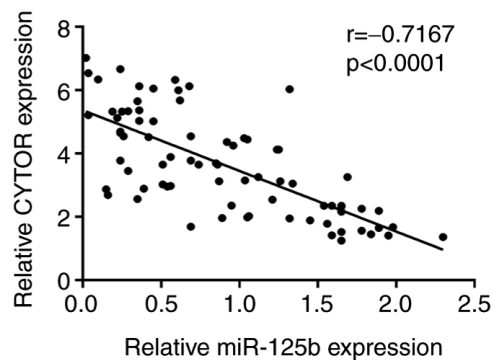

Figure 3. CYTOR directly interacts with miR-125b. (A) Bioinformatics analysis showed a predicted binding site between CYTOR and miR-125b. (B) Transfection efficiency with miR-125b mimic and inhibitor was confirmed by reverse transcription quantitative PCR. (C) Luciferase reporter assay demonstrated that miR-125b overexpression or knockdown could decrease or increase, respectively, the luciferase activity of the CYTOR-WT. (D) RIP assay showed that CYTOR and miR-125b expression were enriched in anti-Ago2 group. (E) RIP assay demonstrated that the enrichment of CYTOR in anti-Ago2 group can be enhanced by miR-125b mimic and inhibited by miR-125b inhibitor. (F) CYTOR negatively regulated miR-125b expression. (G) miR-125b was significantly downregulated in HCC tumor tissues compared with adjacent non-tumor tissues $(\mathrm{n}=78)$. (H) Correlation analysis revealed negative correlation between CYTOR expression and miR-125b expression in HCC tissues $(\mathrm{n}=78) .{ }^{* * *} \mathrm{P}<0.01$ and ${ }^{* * * *} \mathrm{P}<0.001$. CYTOR, cytoskeleton regulator RNA; RT-qPCR, reverse transcription quantitative PCR; NC, negative control; sh, short hairpin; miR, micro-RNA; WT, wild-type; MUT, mutant; RIP, RNA immunoprecipitation.

tive cells in sh-CYTOR\#1 + miR-125b inhibitor group was significantly enhanced compared with that in sh-CYTOR\#1 group (Fig. 4C). In addition, western blotting results showed that the effect of sh-CYTOR\#1 on the expression of the anti-apoptotic protein $\mathrm{Bcl}-2$, and the apoptotic-related proteins, Bax, cleaved-caspase-3, cleaved-caspase- 9 and PARP were partly reversed following co-transfection with miR-125b inhibitor (Fig. 4D). These findings indicated that CYTOR may modulate HCC cell proliferation and apoptosis by sponging miR-125b.
SEMA4C is the target of miR-125b and CYTOR regulates SEMA4C through miR-125b in HCC. The potential target genes of miR-125b were predicted by Targetscan, and SEMA4C was shown to share a binding site with miR-125b (Fig. 5A). The luciferase assay was performed to confirm this prediction and the results demonstrated that the luciferase activity of SEMA4C-WT was significantly decreased in miR-125b mimic-transfected Huh7 cells, which was not the case in SEMA4C-MuT (Fig. 5B), indicating that SEMA4C may be a downstream target gene of miR-125b. 

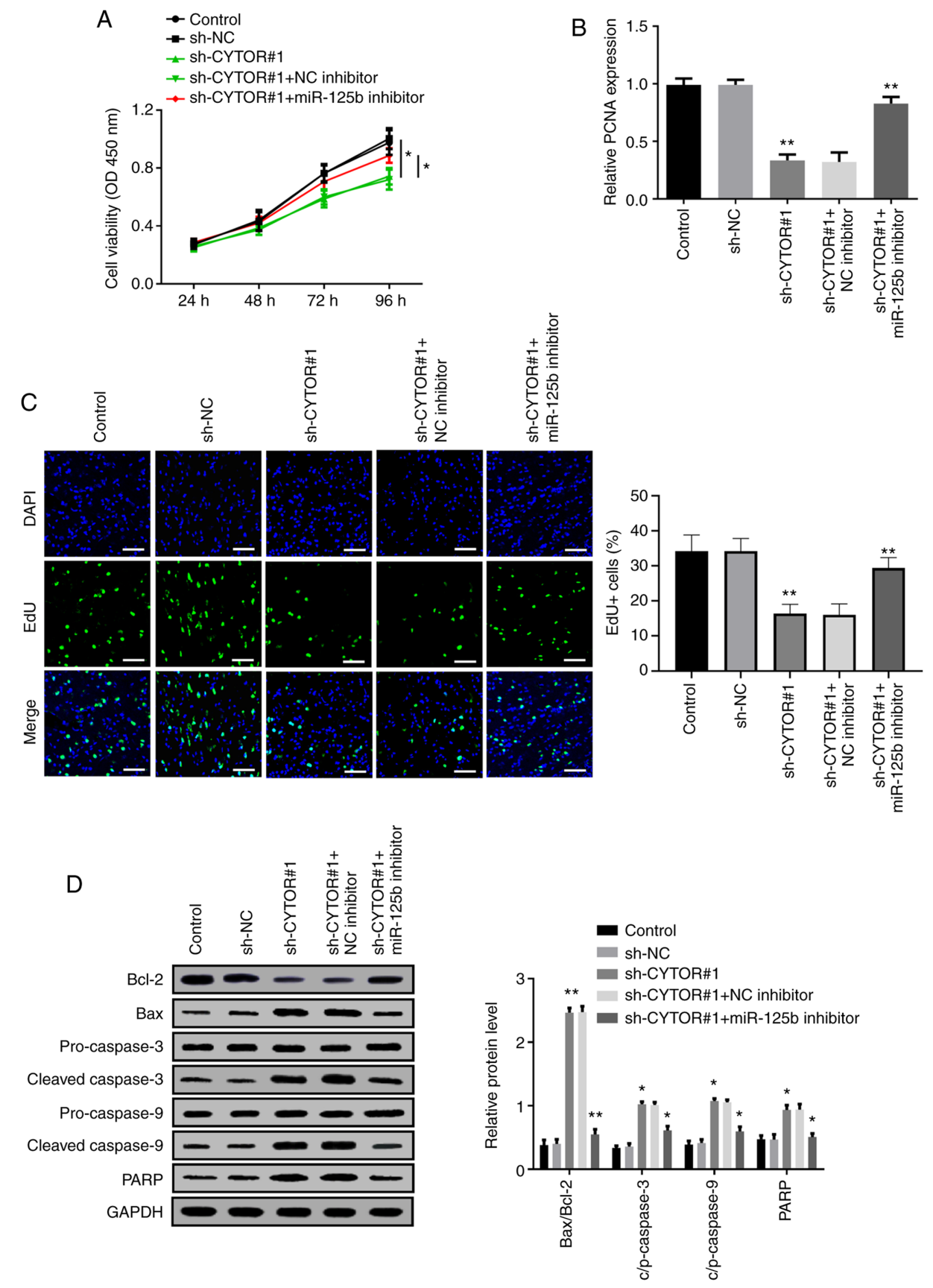

Figure 4. CYTOR sponges miR-125b to modulate HCC cell proliferation and apoptosis. (A) Decreased cell viability induced following sh-CYTOR\#1 transfection was restored by miR-125b inhibitor. (B) Reverse transcription quantitative PCR analysis demonstrated that PCNA expression was decreased by sh-CYTOR\#1 and rescued by miR-125b inhibitor. (C) Suppression of the proliferative effect of sh-CYTOR\#1 was enhanced by miR-125 inhibitor (scale bar, $20 \mu \mathrm{m}$ ). (D) Expression of the apoptosis-related proteins Bcl-2, Bax, cleaved/pro-caspase-3, cleaved/pro-caspase-9 and PARP was determined using western blotting. "P<0.05 and ${ }^{* * *} \mathrm{P}<0.01$. CYTOR, cytoskeleton regulator RNA; RT-qPCR, reverse transcription quantitative PCR; NC, negative control; sh, short hairpin; miR, micro-RNA; OD, optical density; PCNA, proliferating cell nuclear antigen

Furthermore, the results from RT-qPCR and western blotting demonstrated that miR-125b mimic could inhibit SEMA4C expression, whereas miR-125b inhibitor enhanced SEMA4C expression (Fig. 5C and D). Subsequently, the relationship between CYTOR, miR-125b and SEMA4C was investigated, and the results demonstrated that SEMA4C expression decrease by miR-125b mimic was rescued by transfection with pcDNA-CYTOR (Fig. 5E and F), suggesting that CYTOR could regulate SEMA4C expression through targeting miR-125b. SEMA4C expression level in HCC tumor tissues was significantly increased compared with non-tumor tissues (Fig. 5G). The results from Pearson correlation analysis demonstrated that SEMA4C expression was negatively correlated with miR-125b expression; however, SEMA4C expression was positively correlated with CYTOR expression in HCC tissues (Fig. 5H and I). These findings demonstrated that SEMA4C may be a target of miR-125b, and that CYTOR may regulate SEMA4C expression by sponging miR-125b.

CYTOR knockdown inhibits tumor growth in vivo. Huh7 cells transfected with sh-CYTOR\#1 were injected into nude mice to determine the effect of CYTOR on the HCC tumor 


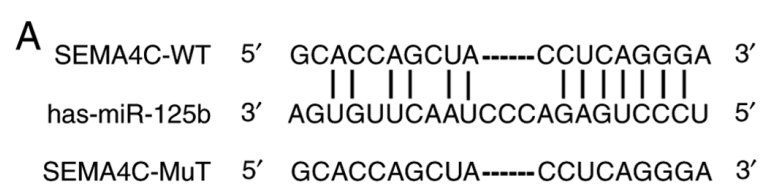

C
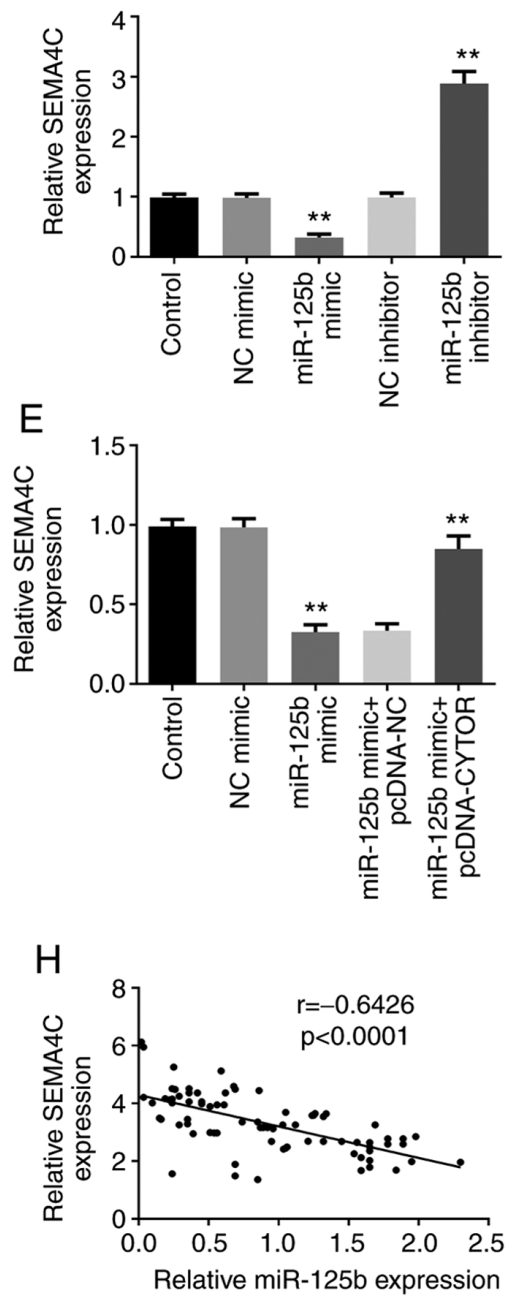
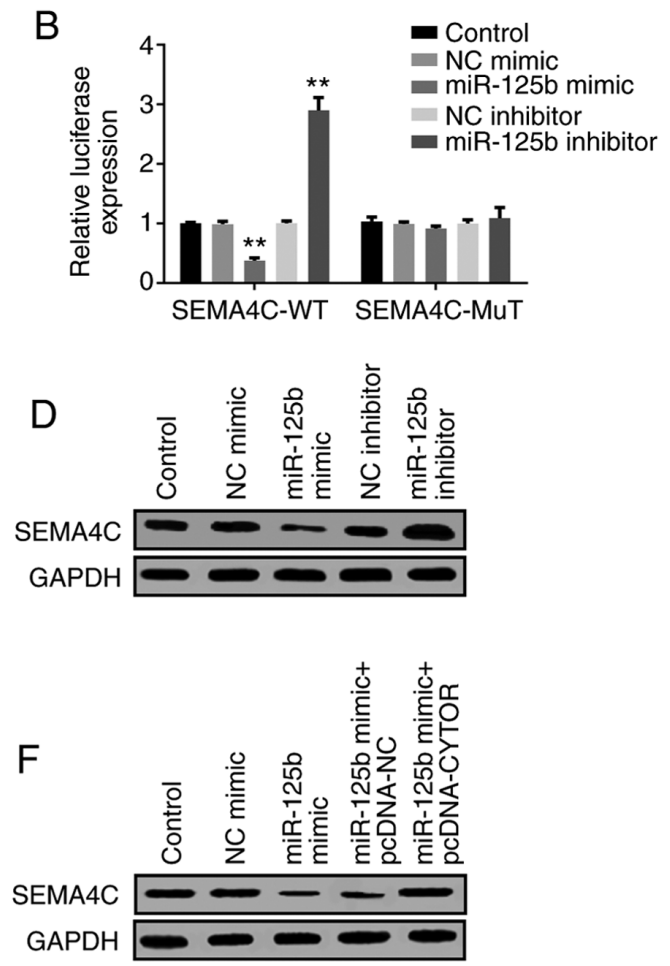

G
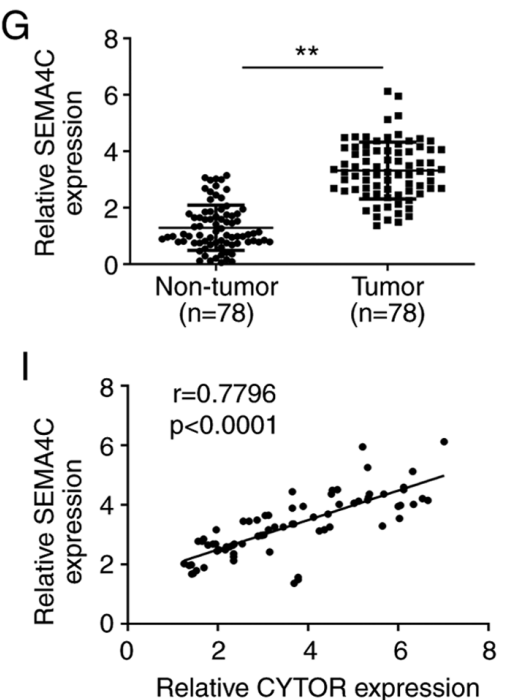

Figure 5. CYTOR regulates SEMA4C expression through miR-125b. (A) Bioinformatic analysis discovered a putative SEMA4C binding site with miR-125b (B) Luciferase activity was detected after co-transfection with miR-125b mimic and luciferase reporters containing WT or Mut SEMA4C transcript (C) SEMA4C mRNA expression and (D) protein expression were decreased following transfection with miR-125b mimic, and increased following transfection with miR-125b inhibitor in Huh7 cells. (E and F) SEMA4C downregulation caused by miR-125b mimic was reversed by CYTOR overexpression. (G) SEMA4C expression was examined by reverse transcription quantitative PCR in HCC tumor and adjacent non-tumor tissues (n=78). (H) Negative correlation was found between SEMA4C expression and miR-125b expression in HCC tissues ( $\mathrm{n}=78$ ). (I) Positive correlation was found between CYTOR expression and SEMA4C expression in HCC tissues $(\mathrm{n}=78) .{ }^{* *} \mathrm{P}<0.01$. CYTOR, cytoskeleton regulator RNA; NC, negative control; sh, short hairpin; miR, micro-RNA; WT, wild-type; MUT, mutant; SEMA4C, semaphorin 4C.

growth. As presented in Fig. 6A and B, the tumor volume in the sh-CYTOR\#1 group grew more slowly during the 28 days observation in comparison with that in the sh-NC group. Furthermore, the tumor weight in the sh-CYTOR\#1 group was significantly decreased compared with control group (Fig. 6C). The result from immunohistochemistry demonstrated that the tumor cell proliferation marker Ki67 and the expression of SEMA4C were decreased in the sh-CYTOR\#1 group compared with the control group (Fig. 6D and E). Furthermore, results from RT-qPCR showed that CYTOR and SEMA4C expression levels were significantly decreased, while miR-125b expression level was significantly increased in tumor tissues of the sh-CYTOR\#1 group compared with tumor tissues of the sh-NC group (Fig. 6F-H). These findings indicated that CYTOR knockdown could inhibit HCC tumor growth in vivo and downregulate SEMA4C expression.

\section{Discussion}

$\mathrm{HCC}$ is the most common type of liver cancer and is characterized by high morbidity and mortality rates worldwide (16). 
A

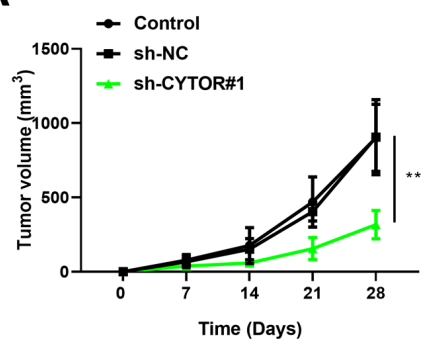

C

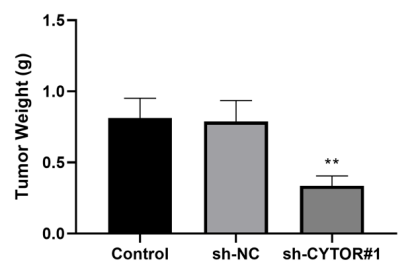

$\mathbf{F}$
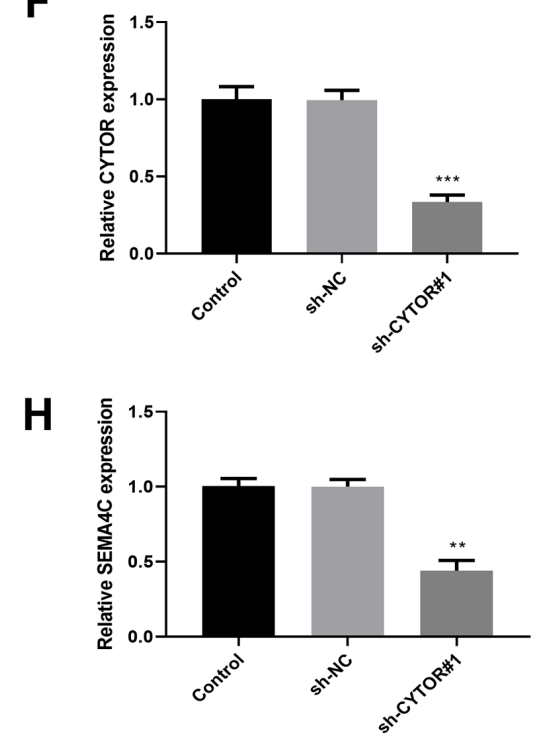

B

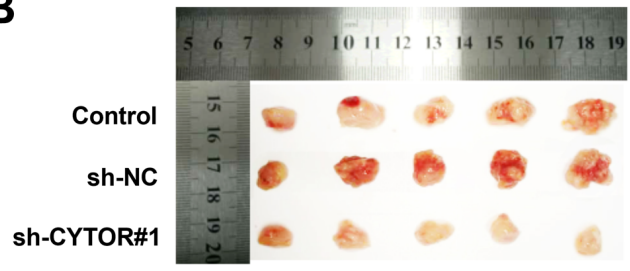

D

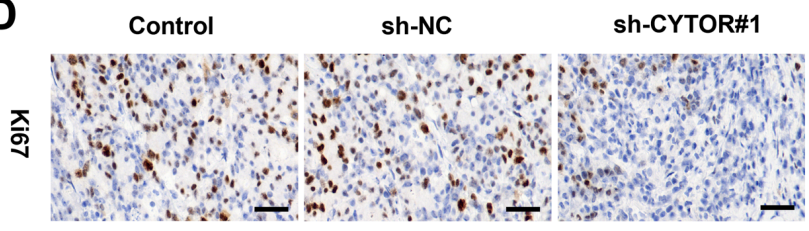

E

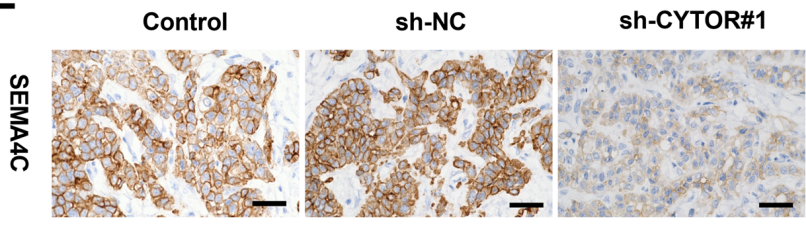

G

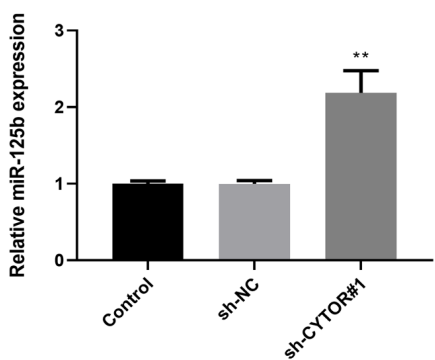

Figure 6. Effect of CYTOR knockdown on tumor growth in vivo. (A) Tumor volume growth curve was detected during the 28 days observation. (B) Images of the tumors isolated from mice. (C) Tumor weight was detected using an electronic scale at 28 days. (D) Expression of Ki67 and (E) SEMA4C in tumor tissues was evaluated by immunohistochemistry (scale bar, $25 \mu \mathrm{m})$. (F) CYTOR, (G) miR-125b and (H) SEMA4C expression levels in tumor tissues were measured by reverse transcription quantitative $\mathrm{PCR} .{ }^{* *} \mathrm{P}<0.01$ and ${ }^{* * *} \mathrm{P}<0.001$. CYTOR, cytoskeleton regulator RNA; NC, negative control; sh, short hairpin; miR, micro-RNA.

Significant progress in understanding the molecular mechanisms of HCC has been made, especially when the competing endogenous RNA hypothesis was presented (17). Currently, research has focused on IncRNAs, which function primarily through interactions with cellular macromolecules, such as proteins and RNAs (18-20). Understanding the molecular mechanism of some lncRNAs in HCC progression is therefore crucial.

The lncRNA CYTOR has been reported to modulate the pathological process of various types of cancer. For example, CYTOR serves an oncogene role in the development of colorectal cancer by interacting with nucleolin and
KH RNA binding domain containing, signal transduction associated 1 (21). CYTOR knockdown was demonstrated to attenuate the migration and invasion of colon cancer cells, while CYTOR overexpression can enhance the metastatic properties of colon cancer cells (22). Liang et al (23) revealed that CYTOR is upregulated in gastric cancer and HCC, and that elevated CYTOR expression is closely associated with the poor prognosis of patients with gastric cancer and HCC. The present study demonstrated that CYTOR was upregulated in HCC tissues and that CYTOR expression was associated with the poor prognosis of patients with HCC. Furthermore, HCC cell proliferation was significantly decreased following 
CYTOR knockdown whereas it was significantly increased by CYTOR overexpression. The levels of apoptotic-related proteins were significantly elevated after CYTOR knockdown, while they were significantly decreased following CYTOR overexpression. Taken together, these findings indicated that CYTOR may serve an oncogenic role in HCC.

Numerous studies have confirmed that IncRNAs play key roles in various types of cancer by sponging miRNAs and consequently regulating gene expression $(24,25)$. In HCC, SOX9-AS1 was reported to stimulate tumor growth and metastasis in HCC through SOX9 and the Wnt/ $\beta$-catenin pathway (8). Furthermore, upregulation of cancer susceptibility 15 (CASC15) can enhance the tumorigenicity and epithelial to mesenchymal transition of HCC by increasing the expression of twist family BHLH transcription factor 1 via sponging to miR-33a-5p (26). Zhang et al (27) reported that the lncRNA $\mathrm{X}$-inactive specific transcript sponges miR-497-5p to promote cell proliferation and migration in HCC. To identify the underlying mechanism of CYTOR in $\mathrm{HCC}$, previous bioinformatics analysis revealed that miR-125b, which was previously discovered as a tumor suppressor in the modulation of HCC progression (28), might be considered as a potential target of CYTOR. The present study reported a negative correlation between CYTOR expression and miR-125b expression, and the results from luciferase reporter assay confirmed that miR-125b may be a direct target of CYTOR. Importantly, miR-125b inhibitor could rescue the inhibitory effect of sh-CYTOR\#1 on HCC cell proliferation and attenuated the promoting effect of sh-CYTOR\#1 on cell apoptosis.

SEMA4C, a member of the semaphorin family, has been reported to be highly expressed in several human cancers and to stimulate the proliferation, migration and invasion of cancer cells $(29,30)$. SEMA4C was previously reported as a functional target of miR-125b in lung cancer (31). The present study demonstrated that miR-125b was bound to the 3'-UTR of SEMA4C and could negatively modulate SEMA4C expression according to the starBase bioinformatics database analysis and the luciferase assay. Previous studies have reported that abnormal expression of SEMA4C exists in various tumors. For instance, SEMA4C expression is significantly elevated in breast cancer and cervical cancer $(32,33)$. Liu et al $(34)$ demonstrated that SEMA4C is upregulated in HCC tissues and promotes tumor growth and cell metastasis in HCC, which is consistent with the results from the present study showing that SEMA4C expression was significantly increased in HCC tissues. The present study also revealed that CYTOR promoted the expression of SEMA4C in HCC cells by decreasing the expression of miR-125b.

This study presented some limitations. Firstly, the relatively small sample size may lead to errors in the analysis of clinicopathological characteristics and overall survival of patients. Secondly, the small number of animals and human observation errors may also lead to statistical errors. Thirdly, the impact of the CYTOR/miR-125b/SEMA4C axis on cell invasion, cell migration and cell cycle, in vitro and in vivo, should be further investigate din the future

In summary, the findings form the present study demonstrated that CYTOR was highly expressed in HCC tissues and cells and could stimulate HCC cell proliferation and tumor growth via modulating the miR-125b/SEMA4C axis. The CYTOR/miR-125b/SEMA4C axis may therefore provide a new perspective for the development of novel therapeutic strategy for HCC.

\section{Acknowledgements}

Not applicable.

\section{Funding}

This study was supported by the Tianjin Science and technology plan project (grant no. 19ZXDBSY00010).

\section{Availability of data and materials}

The datasets used and/or analyzed during the present study are available from the corresponding author on reasonable request.

\section{Authors' contributions}

QT performed the experiements, guaranteed the integrity of the entire study and participated in writing the manuscript. XY, LY, ZL and ZY collected the clinical samples, performed the data analysis and statistical analysis. YZ designed the study, supervised the research and participated in the writing and reviewing of the manuscript. All authors read and approved the final manuscript. QT and YZ confirmed the authenticity of all the raw data.

\section{Ethics approval and consent to participate}

The experiments using human samples were approved by the Clinical Research Ethics Committee of Tianjin First Central Hospital (approval no. 2019N013YY). Signed written informed consents were obtained from the patients and/or guardians. The animal protocol was approved by the Animal Care and Use Committee (approval no. 2019N046YY).

\section{Patient consent for publication}

Not applicable.

\section{Competing interests}

The authors declare that they have no competing interests.

\section{References}

1. Siegel R, Naishadham D and Jemal A: Cancer statistics, 2012. CA Cancer J Clin 62: 10-29, 2012.

2. Michelotti GA, Machado MV and Diehl AM: NAFLD, NASH and liver cancer. Nat Rev Gastroenterol Hepatol 10: 656-665, 2013.

3. Yu JJ, Xiao W, Dong SL, Liang HF, Zhang ZW, Zhang BX, Huang ZY, Chen YF, Zhang WG, Luo HP, et al: Effect of surgical liver resection on circulating tumor cells in patients with hepatocellular carcinoma. BMC Cancer 18: 835, 2018.

4. Hu X, Jiang J, Xu Q, Ni C, Yang L and Huang D: A systematic review of long noncoding RNAs in hepatocellular carcinoma: molecular mechanism and clinical implications. BioMed Res Int 2018: 8126208, 2018. 
5. Bhan A, Soleimani M, Mandal SS and Long Noncoding RNA Long noncoding RNA and cancer: A new paradigm. Cancer Res 77: 3965-3981, 2017.

6. Yang X, Yao B, Niu Y, Chen T, Mo H, Wang L, Guo C and Yao D: Hypoxia-induced lncRNA EIF3J-AS1 accelerates hepatocellular carcinoma progression via targeting miR-122-5p/CTNND2 axis. Biochem Biophys Res Commun 518: 239-245, 2019.

7. Feng J, Yang G, Liu Y, Gao Y, Zhao M, Bu Y, Yuan H, Yuan Y, Yun $\mathrm{H}$, Sun M, et al: LncRNA PCNAP1 modulates hepatitis B virus replication and enhances tumor growth of liver cancer. Theranostics 9: 5227-5245, 2019.

8. Zhang W, Wu Y, Hou B, Wang Y, Deng D, Fu Z and Xu Z: A SOX9-AS1/miR-5590-3p/SOX9 positive feedback loop drives tumor growth and metastasis in hepatocellular carcinoma through the Wnt/ $\beta$-catenin pathway. Mol Oncol 13: 2194-2210, 2019.

9. Ji D, Hu G, Zhang X, Yu T and Yang J: Long non-coding RNA DSCAM-AS1 accelerates the progression of hepatocellular carcinoma via sponging miR-338-3p. Am J Transl Res 11: 4290-4302, 2019.

10. Pang Q, Ge J, Shao Y, Sun W, Song H, Xia T, Xiao B and Guo J: Increased expression of long intergenic non-coding RNA LINC00152 in gastric cancer and its clinical significance. Tumour Biol 35: 5441-5447, 2014

11. Li M, Wang Q, Xue F and Wu Y: lncRNA-CYTOR Works as an oncogene through the CYTOR/miR-3679-5p/MACC1 axis in colorectal cancer. DNA Cell Biol 38: 572-582, 2019.

12. Liu Y, Li M, Yu H and Piao H: IncRNA CYTOR promotes tamoxifen resistance in breast cancer cells via sponging miR 125a 5p. Int J Mol Med 45: 497-509, 2020.

13. Zhang $\mathrm{J}$ and $\mathrm{Li} \mathrm{W}$ : Long noncoding RNA CYTOR sponges miR-195 to modulate proliferation, migration, invasion and radiosensitivity in nonsmall cell lung cancer cells. Biosci Rep 38: 38, 2018.

14. Thuluvath PJ, To C and Amjad W: Role of locoregional therapies in patients with hepatocellular cancer awaiting liver transplantation. Am J Gastroenterol 116: 57-67, 2021.

15. Livak KJ and Schmittgen TD: Analysis of relative gene expression data using real-time quantitative PCR and the 2(-Delta Delta C(T)) method. Methods 25: 402-408, 2001

16. Ruggieri A, Barbati C and Malorni W: Cellular and molecular mechanisms involved in hepatocellular carcinoma gender disparity. Int J Cancer 127: 499-504, 2010.

17. Salmena L, Poliseno L, Tay Y, Kats L and Pandolfi PP: A ceRNA hypothesis: The Rosetta Stone of a hidden RNA language? Cell 146: 353-358, 2011.

18. Wang KC and Chang HY: Molecular mechanisms of long noncoding RNAs. Mol Cell 43: 904-914, 2011.

19. Böhmdorfer $\mathrm{G}$ and Wierzbicki AT: Control of chromatin structure by long noncoding RNA. Trends Cell Biol 25: 623-632, 2015.

20. Bonasio R and Shiekhattar R: Regulation of transcription by long noncoding RNAs. Annu Rev Genet 48: 433-455, 2014

21. Wang X, Yu H, Sun W, Kong J, Zhang L, Tang J, Wang J, Xu E, Lai $\mathrm{M}$ and Zhang $\mathrm{H}$ : The long non-coding RNA CYTOR drives colorectal cancer progression by interacting with NCL and Sam68. Mol Cancer 17: 110, 2018.
22. Yue B, Liu C, Sun H, Liu M, Song C, Cui R, Qiu S and Zhong M: A Positive Feed-Forward Loop between LncRNA-CYTOR and Wnt $/ \beta$-Catenin Signaling Promotes Metastasis of Colon Cancer. Mol Ther 26: 1287-1298, 2018.

23. Liang J, Wei X, Liu Z, Cao D, Tang Y, Zou Z, Zhou C and Lu Y: Long noncoding RNA CYTOR in cancer: A TCGA data review. Clin Chim Acta 483: 227-233, 2018.

24. Wang H, Huo X, Yang XR, He J, Cheng L, Wang N, Deng X, Jin $\mathrm{H}$, Wang $\mathrm{N}$, Wang $\mathrm{C}$, et al: STAT3-mediated upregulation of IncRNA HOXD-AS1 as a ceRNA facilitates liver cancer metastasis by regulating SOX4. Mol Cancer 16: 136, 2017.

25. Li SP, Xu HX, Yu Y, He JD, Wang Z, Xu YJ, Wang CY, Zhang HM, Zhang RX, Zhang JJ, et al: LncRNA HULC enhances epithelial-mesenchymal transition to promote tumorigenesis and metastasis of hepatocellular carcinoma via the miR-200a-3p/ ZEB1 signaling pathway. Oncotarget 7: 42431-42446, 2016.

26. Li Y, Chen G, Yan Y and Fan Q: CASC15 promotes epithelial to mesenchymal transition and facilitates malignancy of hepatocellular carcinoma cells by increasing TWIST1 gene expression via miR-33a-5p sponging. Eur J Pharmacol 860: 172589, 2019.

27. Zhang Y, Zhu Z, Huang S, Zhao Q, Huang C, Tang Y, Sun C, Zhang Z, Wang L, Chen H, et al: lncRNA XIST regulates proliferation and migration of hepatocellular carcinoma cells by acting as miR-497-5p molecular sponge and targeting PDCD4. Cancer Cell Int 19: 198, 2019

28. Hua S, Quan Y, Zhan M, Liao H, Li Y and Lu L: miR-125b-5p inhibits cell proliferation, migration, and invasion in hepatocellular carcinoma via targeting TXNRD1. Cancer Cell Int 19: 203, 2019.

29. Li J, Wang Q, Wen R, Liang J, Zhong X, Yang W, Su D and Tang J: MiR-138 inhibits cell proliferation and reverses epithelial-mesenchymal transition in non-small cell lung cancer cells by targeting GIT1 and SEMA4C. J Cell Mol Med 19: 2793-2805, 2015.

30. Yang Q, Wang Y, Lu X, Zhao Z, Zhu L, Chen S, Wu Q, Chen C and Wang Z: MiR-125b regulates epithelial-mesenchymal transition via targeting Sema4C in paclitaxel-resistant breast cancer cells. Oncotarget 6: 3268-3279, 2015.

31. Zhang Y and Huang S: Up-regulation of miR-125b reverses epithelial-mesenchymal transition in paclitaxel-resistant lung cancer cells. Biol Chem: Aug 20, 2015 (Epub ahead of print). doi: 10.1515/hsz-2015-0153.

32. Yang J, Zeng Z, Qiao L, Jiang X, Ma J, Wang J, Ye S, Ma Q, Wei J, Wu M, et al: Semaphorin 4C Promotes macrophage recruitment and angiogenesis in breast cancer. Mol Cancer Res 17: 2015-2028, 2019.

33. Song J and Li Y: miR-25-3p reverses epithelial-mesenchymal transition via targeting Sema4C in cisplatin-resistance cervical cancer cells. Cancer Sci 108: 23-31, 2017.

34. Lu J, Lin Y, Li F, Ye H, Zhou R, Jin Y, Li B, Xiong X and Cheng N: MiR-205 suppresses tumor growth, invasion, and epithelial-mesenchymal transition by targeting SEMA4C in hepatocellular carcinoma. FASEB J: May 25, 2018. doi: 10.1096/fj.201800113R. 